\title{
JHK PHOTOMETRY OF EXTRAGALACTIC SOURCES USING AN INFRARED
} ARRAY CAMERA

\author{
I. S. McLean, T. Liu and H. Teplitz \\ Department of Physics and Astronomy, UCLA
}

\begin{abstract}
The UCLA two-channel IR camera has been used to perform crowded-field photometry in two distinct classes of extragalactic objects. DAOPHOT was used to reduce images of M 33 and provide JHK color-magnitude diagrams of over 1,700 stars. Galaxy photometry in crowded clusters, e.g. Abell 370, was obtained in JHK and analyzed using FOCAS. In both cases, extensive reductions were performed using synthetic aperture photometry for comparison. Methods and results are summarized.
\end{abstract}

\section{INTRODUCTION}

The development of infrared array detectors for astronomy has had an enormous impact (see McLean 1994 for reviews). These detectors are now sufficiently large ( 256 x 256 pixels) and sensitive $(\mathrm{QE}>60 \%$; read noise $<50$ electrons) to find a wide range of photometric applications. Two examples are used to illustrate both the performance and the potential of IR arrays: JHK photometry of the core of the Local Group galaxy M 33 and JHK photometry for two galaxy clusters (Abell 370 and CL0024+1654). Full details of the two-channel camera used are given in McLean et al. (1993) and McLean et al. (1994).

\section{OBSERVATIONS OF M 33}

The observations were made in two sets; (J, K') in 10/93 and (H, $\left.K^{\prime}\right)$ in $12 / 93$; $K^{\prime}$ refers to the Mauna Kea K-filter (1.95 - 2.30 microns). A three by three overlapping mosaic was used to obtain a field of $7.6 \times 7.6$ arc minutes with total integration times of $315 \mathrm{~s}$ at J, $300 \mathrm{~s}$ at $\mathrm{H}$ and $300 \mathrm{~s}$ at $\mathrm{K}^{\prime}$. The $\mathrm{K}^{\prime}$ frames from October and December were compared but not combined. Conversion to standard $\mathrm{K}$ magnitudes was done using the relationship given by Wainscoat and Cowie (1992). Raw frames at each wavelength were first processed by subtracting a sky frame constructed from the median of nearby empty sky fields and then divided by a dark-subtracted and normalized dome flat field.

Using DAOPHOT, over 1,700 stars were measured reliably. The uncertainties in the photometry are as follows:

$\mathrm{J}: 0.17 \mathrm{mag}$ at $\mathrm{J}=16$ to $0.70 \mathrm{mag}$ at $\mathrm{J}=19$

$\mathrm{H}: 0.23 \mathrm{mag}$ at $\mathrm{H}=16$ to $0.50 \mathrm{mag}$ at $\mathrm{H}=18$

$\mathrm{K}: 0.27$ mag at $\mathrm{K}=16$ to 0.53 mag at $\mathrm{K}=18$.

The DAOPHOT artificial star experiment was used to determine these error estimates and the completeness limits by recovering "fake" stars. 
Photometry was performed on the final, registered mosaic using an average PSF determined from various positions in the mosaic after careful comparison of results obtained with locally determined PSFs showed excellent agreement. Aperture photometry was applied to the bright and less crowded stars to calibrate the PSF photometry.

Color-magnitude diagrams and luminosity functions have been derived. A well-defined giant branch is seen which is brighter than globular clusters and Baade's Window giants in our galaxy, but similar to M 31 and M 32. The fraction of luminous stars seems enhanced over that of $M 31$ and M 32. Based on the large number of $O B$ associations in M 33, the brightest stars are probably supergiants from recent star formation.

\section{OBSERVATIONS OF ABELL 370 AND CLOO24+1654}

JHK observations of these two clusters of galaxies at $z=0.37$ were obtained on October 24 and 25, 1993. The $K^{\prime}$ ' band was observed continuously in the long wave channel, with the $\mathrm{J}$ and $\mathrm{H}$ observations "nested" within the total $\mathrm{K}$ ' integration period. For CLO024 the K':H:J exposure times in minutes were 135:90:45 and for Abell 370 the integration times were 90:45:45. Observations were made in five minute segments and the telescope was dis-registered or dithered between each integration. A running median "sky flat" was derived from the cluster frames themselves and applied to each frame in turn. All the flatfielded frames were then registered and a median of these was formed.

Since most of the objects are slightly extended, identification was done using the FOCAS software package which produces a complete list of objects found. Synthetic aperture photometry was performed using a standard aperture size of 4.2 arc seconds for each object. Completeness was estimated by using IRAF routines to introduce "fake" stars into the frames and recover them. For $\mathrm{Cl} 0024+1654$ the limit is $\mathrm{K}^{\prime}=18.6$ (five sigma) and for Abell 370 it is 17.9. Over 150 galaxies are found.

(J-K)' versus K' color-magnitude diagrams were formed for both clusters. Interestingly, both clusters exhibit gravitational lensing arcs. JHK photometry for Arclets A1, A3 and A4 in CL0024+16 and arcs A5 and 62 in Abell 370 reveals that all three arclets in CL0024+16 have approximately similar colors, with the average value of $\left(\mathrm{J}-\mathrm{K}^{\prime}\right)=1.33$ (range is $1.2-1.5$ ), whereas for Abell 370 the (J-K') colors are 1.4 and 2.0 for arcs 62 and A5 respectively. Since both of the arcs in Abell 370 have known redshifts of $z=0.7$ and $z=1.3$, it is plausible that all three arclets in CL0024+16 come from the same background galaxy at approximately $z=1$.

\section{REFERENCES}

McLean, I. S., Becklin, E. E., Brims, G., Canfield, J., Casement, L. S., Figer, D. F., Henriquez, F., Huang, A., Liu, T., Macintosh, B. and Teplitz, H. 1993 Proc. SPIE 1946, Infrared Detectors and Instrumentation, A. M. Fowler, ed., p. 513

McLean, I. S., Macintosh, B., Liu, T., Casement, L. S., Figer, D. F., Teplitz, H., Larson, S., Lacayanga, F., Silverstone, M. and Becklin, E. E. 1994 Proc. SPIE 2198, Instrumentation in Astronomy VI, D. L. Crawford and E. R. Craine, p. 457

McLean, I. S., ed. 1994 Infrared Astronomy with Arrays: The Next Generation, Kluwer Academic Publishers, Dordrecht

Wainscoat, R. J. and Cowie, L. L. 1992 AJ 103, 332 